\title{
BEYOND INSTRUCTIONAL DESIGN: MAKING LEARNING DESIGN A REALITY
}

\author{
Rod Sims \\ Capella University \\ rod.sims@faculty.capella.edu
}

\section{Introduction}

When we reflect on the emergence of online education and e-learning as the leading contender to confront the traditions of face-to-face teaching and learning, it is not only a case of better understanding the characteristics of online environments, but also timely to assess the relevance of theories and frameworks informing the design and implementation of those environments. Over the past twenty five years, the value of technology to education has been a significant focus of teachers, learners and institutional administration; it is also a period that has been characterised by lighthouse success stories, rigorous research, technological determinism and unfulfilled promises. As each new generation of technology appeared in the classroom (microcomputers, colour monitors, hypermedia, multimedia, internet), a new generation of early adopters appeared, each seemingly unaware of the research and knowledge gained by the previous generation. In this paper the argument is proposed that even with the strong foundation of knowledge that informs the appropriate ways to use technology for teaching and learning, too often that knowledge has been misunderstood or misused or ignored.

The paper is based on the keynote presentation to the Online Learning and Teaching Conference held in Brisbane on September $23^{\text {rd }}$ 2005. The theme of the conference, Beyond Delivery, was extremely timely as it marked the assertion that it was time to put the simple digitisation of materials behind us and focus on the active, engaging learning opportunities that effectively put the learning back into e-learning to take advantage of collaborative tools, learning communities and mediated conversation for new millennial learners. More importantly the statement that we are beyond delivery also suggests that it is time to embrace change and reflect on new ways to conceptualise the design of online learning environments.

Through this paper an argument is presented for a proactive approach to the conceptualisation, formation and maintenance of online environments that cater specifically for the individual learner. Through an analysis of design strategies, proactive modelling and interactive metrics, a new framework is presented to address the pedagogy of online environments and present an alternative to common instructional design practices. For those committed to online teaching and learning environments populated with collaborating and reflective participants, this framework goes well beyond delivery to a pedagogy centred on emancipation and empowerment for the engaged learner.

\section{Design Strategies}

\section{Instructional Design and Epistemology}

When we consider practitioner understandings of Instructional Design, the methods and processes often reduce to the components of Analysis, Design, Development, Implementation and Evaluation (ADDIE), where one stage follows another in a linear sequence. Within this context, the following quotes highlight the designer-centred aspect of both instruction and design: 
Instruction ... an instruction is a form of communicated information ... for how an action, behavior, method, or task is to be begun, completed, conducted, or executed (Wikipedia, 2006a)

Design ... the process of originating and developing a plan for aesthetic and functional objects ... requiring considerable research, thought, modelling, iterative adjustment and re-design ... (Wikipedia, 2006b)

Instructional Design ... the analysis of learning needs and systematic development of instruction ... that if followed, will facilitate the transfer of knowledge, skills and attitude to the recipient or acquirer of the instruction ... (Wikipedia, 2006c)

Using these descriptors, and to emphasise the position taken in this paper, there is a sense that Instructional Design focuses on following pre-determined pathways that, if undertaken rigorously, will ensure a transfer of knowledge. But is education about the transfer of knowledge, or does it involve more complex processes between teachers, learners and the content domain? Those with an interpretivist epistemology (Driscoll, 2005) would argue that knowledge is constructed by the individual and therefore goes beyond an information transmission model. Based on over 25 years of working with and researching the use of computers in a learning and performance context, it is my contention that computers should preferably be used for course participants to deconstruct, construct or reconstruct their mental models and that information transmission (delivery) strategies do not make effective use of computer technology. The computer, whether being used independently or for online communications, is ideal for supporting a constructivist approach to education.

With these considerations in mind I would therefore propose a conditional statement:

if instruction represents a form of delivery, and

if we are beyond delivery, then

we have reached a stage where we are beyond instruction.

Consequently we need to address different models of teaching and learning that build upon the foundations of instructional design and yet transcend its epistemological foundations, that are often based on objectivist (knowledge is external) principals (Driscoll, 2005). One of the significant reasons for this is that the learners who are now entering formal education potentially have quite different skill sets than those for whom instructional design was devised.

\section{New Millenial Learners}

In considering why online education needs to go beyond delivery, we need to consider both the pedagogy of the online environment and the diverse participants who populate that environment. Ulmer (2003) introduced the concept of electracy, an extension from the Ancient Greek invention of literacy, designed to explain and bring today's students into the process of invention, a process which, Ulmer argues, includes both emancipation and empowerment. Interestingly, when we assess the system diagrams of online learning software we often see extensive detail of the technology components (learning management system, learning object repositories, content management systems) with the user portrayed as a singular, homogenous entity. If indeed learners are (or will be) empowered and emancipated then we need learning environment representations to portray the system as a single, transparent, multifunctional application and the user (learner, teacher and/or designer) as the creator and definer of the environment (e.g. Prensky, 2005).

More specifically, Prensky argued that today’s learners need to be engaged or they will become enraged, and that the solution may be revealed through game-based curricula. In arguing for such an environment, Prensky identified a range of phrases and actions ...

Create your own heroes ... Encounter ... Engage ... Fly ... Explore ...

Challenge ... Master ... Amass ... Build ... Perform ... Research ... Lead ...

Don't work alone ... 
... concluding that these terms were not exactly descriptions of today's classrooms and courses.

Based on these perspectives of the new generation of learners, we are faced with a design conundrum where instructional design epistemological traditions may not be consistent with the constructivist, collaborative engagements afforded by online environments. At the same time we have the potential of a new generation of learners for whom technology IS the environment and for whom learning means different things. If we adopt this assumption, then we must re-think the paradigms for conceptualising, creating and implementing online learning environments.

In the following section, a proactive set of strategies are summarised that are designed to reconceptualise the instructional design frameworks to maximise the benefits of online education. This is followed by an exposition of interaction metrics designed to extend the ways we can maximise the interactive benefits of online teaching and learning.

\section{Proactive Modelling}

\section{Proactive Design}

The issue with design is that too often content is considered the primary focus and used to define the structure of the course - a legacy of instructional design processes that divide content into hierarchies, thereby providing the framework for menu systems for learners to access content for delivery. However, this approach counters the potential of computer-based environments to provide engaging, interactive and collaborative activities. Based on the matrices proposed by Sims, Dobbs and Hand (2002), the origin of the content (from teacher prescribed to learner generated) and the design of the interface (non-contextual to integrated narrative) are keys to the success of online environments.

With respect to the content aligned to a course, how valuable might strategies be where learners are the proactive providers of content, not just recipients? And with the interface, how effective is a narrative approach, where the learner is integrated into a scripted journey? Through activities designed to engage with key elements of the course content, the learner becomes integrated with a situated and contextualised environment, providing them with a level of control over that learning environment. Rethinking the way we approach factors such as course content and learnercomputer interface also supports the shift from teacher-centred to learner-centred environments (Reigeluth, 1999), which also demands a reappraisal of the roles all participants play in the educational process.

\section{Proactive Roles and Presence}

In recent publications (Sims \& Jones, 2003; Sims \& Hedberg, in press) we have argued that the roles of all participants in the educational process - administrators, technicians, designers, teachers and learners - need to be reassessed to understand their interrelationship within the online context. In the face-to-face environments that are familiar to many, the design of a program or course may take place quite independently from its delivery. However in the online context, design can be something teachers and learners can also participate in, blurring what were once separate roles.

The roles once seen as independent (teacher, learner, designer) now need to be understood as interchangeable. The teacher may need to take on the role of a learner when a learner introduces new content with which the teacher is unfamiliar; a designer may take on the role of teacher to develop a better idea of the impact of their design strategies; the learner may become a teacher or designer as the technology affords opportunities to adapt and change both content, learning strategies and context as the course progresses (much the same way as face-to-face classes can be modified on the fly).

These roles also impact on the way we virtually present ourselves within online teaching and learning environments. While the focus of presence research (e.g. Rourke, Anderson, Garrison \& 
Archer, 1999) has examined the ways in which the interactions of the face-to-face environment can be established online, I would argue that experienced online teachers and learners are developing their own skills of communication, and it is no longer a case that we need the familiar visual cues to determine how teachers or learners are performing and how to enhance reflective practice (Sims \& Bovard, in press).

The rise of online education has changed the way we can teach and learn. It is not just a matter of taking existing strategies to the networked communities, but an imperative to rethink, reshape and redefine what it means to teach and learn in the online world. Given this, it is essential to better understand how to implement and evaluate the effectiveness of the interactions in computermediated learning environments.

\section{The Challenge Of Effective Interactivity}

One of the key attributes of computer-mediated environments is the explicit interaction between the learner and the course content, the learner and other course participants (including the teacher) and the learner and the computer interface (Moore, 1989; Wagner, 1994; Sims, 1997a, 1997b; 1999). In conjunction these three modes of interaction also imply an implicit interaction between the learner and the course designer (Sims \& Hedberg, in press). However, too often the potential of interaction to establish engagement with the content and construction of meaning has been ignored (Sims, 2003).

Given this, the following discussion introduces seven metrics that can be used by online participants to assess the extent to which the interactions in a course of study will effectively engage the learner with the purpose and value of the course outcomes. These metrics have been formulated over the past two decades through my work in designing, developing and teaching; they are best used as a set of heuristics that will maximise the likelihood of an online course achieving both the learning outcomes and providing an engaging learning experience. In presenting these metrics it is important to note that some may be more relevant to self-paced rather than collaborative environments; however their importance lies in enhancing the interactive dynamics of online education. To provide some guidance I have indicated in brackets where the metric is more relevant to Self-Paced (SP) or Collaborative Online (CO) environments.

\section{Metric 1: Learning Style (SP, CO)}

Too often we hear about courses designed to cater for the different learning or cognitive styles of learners. While it is clear we have certain preferences for how we learn, it is not practical or realistic for a designer to predict what particular style a learner might use within a course of study. However, it is feasible for the designer, who has a comprehensive understanding of the different ways people do learn, to implement a series of strategies and resources whereby the learner can apply whichever style they wish to use at the time. This highlights a shift that designers need to make - one where empowerment is with the learner and where the learner is in control of their learning. It is not a case of designing for an assumed learner predisposition, but ensuring that the learner is able to use their personal preferences to contextualise their experience.

An associated issue is the increasing demand for courses that can be delivered on a global basis and to learners from diverse cultures. Again, it is not for the designer to try and design for a specific culture, as that is likely to be fraught with assumptions. Instead, the design must allow for the learner to adapt and contextualise the content and outcomes to their own environment. In making these points it is also pertinent to highlight that culture (and within that, the complexities of elements such as gender or class) can vary across a city as much as it varies between countries and nationalities. Empowering the learner to make decisions based on their own cultural background and cognitive preferences is essential.

Metric 2: Course Completion (SP)

At any point in a course, what is the learner doing and why are they doing it? I have evaluated many self-paced courses where, if they chose, learners could complete that course by simply 
pressing the Enter key repeatedly, and with their eyes closed. Such courses manifest poor design and interactivity, despite often having visually pleasing interfaces and media resources. For a course to be truly interactive, the learner must not be able to complete the course unless they have successfully undertaken an activity or problem that allows them to proceed to the next stage. In addition, self-paced courses need to have a variety of paths that the learner may travel - the content need not be identical for all.

This factor links to the underpinning design philosophy. A traditional model might be typified where the course is delivered and the learner tested to ascertain their level of mastery. However, this effectively places control with the teacher and designer, not the learner. The alternative is to design with a narrative-based sequence (as discussed above) such that completion of the course is in itself an indication of success and mastery. Any testing is built into the narrative and activities of the course. Even if there are certain professional certification requirements for testing, these too can be built into the course.

\section{Metric 3: Cognitive Activity (SP, CO)}

What is the learner actually doing when responding to a discussion forum or working through a self-paced multimedia learning object? It often seems that the rhetoric of active learning we hear from so many designers is manifested in quality graphics but passive, digital paper. Regardless of the developmental stage of the learner or their field of study, it is essential for them to be actively engaged with the environment - assuming that critical thinking in a flexible, complex and global environment is the primary target. This engagement can only be achieved if course participants have the opportunity to test assumptions (hypothesise), construct solutions (manipulate), adjust variables (experiment) and/or introduce content (modify) within that environment. By promoting cognitive activity and engagement (Kennedy, 2004), the learner will be consciously engaged with the interplay between content, context and situation. A useful strategy when reviewing interactive applications with this metric in mind is to ask, at every display, "what is the learner doing here"?

\section{Metric 4: Roles (SP, CO)}

One useful way to approach design is to use the metaphor of theatre (Laurel, 1991). Using this concept, designers can consider the learner as an actor immersed within a particular environment and working with a script, but one where the learner has the opportunity to rehearse and improvise with their fellow course participants. At the same time, it is also critical that the designer effectively communicate the rationale underpinning the design to the learner - too often that is defined through course objectives that lack meaning and relevance.

Importantly, we are reaching a stage where we can no longer differentiate the roles of designer, teacher and learner in a simple way (Sims \& Jones, 2003). For example, while retaining their role in the environment, teachers must remain open to receiving content into the course from learners who have specific knowledge or experience. In this way the presence and roles (considered previously) can be flexible and open to change. Rather than assuming the roles that will be played, it is important for all participants in the online learning environment to be able to articulate and negotiate their preferred roles.

\section{Metric 5: Audio/Visual Effects (SP)}

The media elements that we use in the creation of media-rich environments can either enhance or compromise the learning experience. At all times, each media element needs to be assessed for the extent to which it will enhance the means by which the learner can effectively engage with the content. Otherwise there is a high probability that certain media elements will interfere with that engagement and disrupt the narrative. Media should never be used to demonstrate the software skills of the development team!

One of the reasons this is important is that learners are typically (although not always) excluded from the design process and the design team will make decisions about what they think the learner will like. However in my experience we cannot make this assumption. Linking back to Metric 1 on Learning Styles and Preferences, we should as far as possible allow the learner to make decisions about the presentation of the course. One example I witnessed was in a major CD 
project where a range of sounds were integrated as learners navigated the information. A learner made a choice to see more detail about Thailand and a short musical track was played; the learner immediately commented that the sound was not Thai music and decided that the remaining information would not be useful.

While a simple example, we cannot predict how learners will react. With it becoming easier to integrate animations, audio and video we, as designers, need to take more and more care in when and how to use those media elements.

\section{Metric 6: Feedback (SP, CO)}

Feedback has been identified as a key element of all learning endeavours (Sims, 2003; Driscoll, 2005). Designers must therefore enable a feedback system that is contextual and adaptive as well as positive and correctional (where appropriate). This means that learners will experience feedback as individualised and not as something generic and similar for all (Sims, 2003). While this changes how teachers might communicate with learners, the feedback loop in the online environment is critical to its success.

Working with diverse online learners, one established strategy that can maximise the personalisation of feedback is self-assessment and self-evaluation, integrating reflective practice (Laurillard, 1993) and reflective interactivity (Sims, 1997b). A key success factor in this mode is to ensure that learners are comfortable with being empowered in the learning process, which requires significant effort in developing their model of independence and ensuring they have the course expectations well articulated. Interestingly, the emergence of the independent, flexible learner is in stark contrast to the standards and testing regime being adopted by many educational administrations.

\section{Metric 7: Design for Learning (SP, CO)}

Overall these elements of interaction must be integrated with delivery, content, strategy and interface, as represented in Figure 1. While this model can be aligned to many of the Instructional Design paradigms (Reigeluth, 1999), we need to repurpose current design and development models to emphasise and acknowledge the role of the learner and to embrace the shift to a learnercentred focus.

For example, we often see the roles associated with design as content or institutionally centred instructional designers, project managers, graphic artists, network specialists. However where are the designers for learning or the interactive architects? Where are the collaborative environment specialists? We operate in a learner-centred collaborative context and yet our design models are too often predicated on face-to-face and teacher-centred paradigms. If we are to achieve the full potential and benefits that an online environment affords then we must rethink the philosophies and practices that we bring to the design environment. 


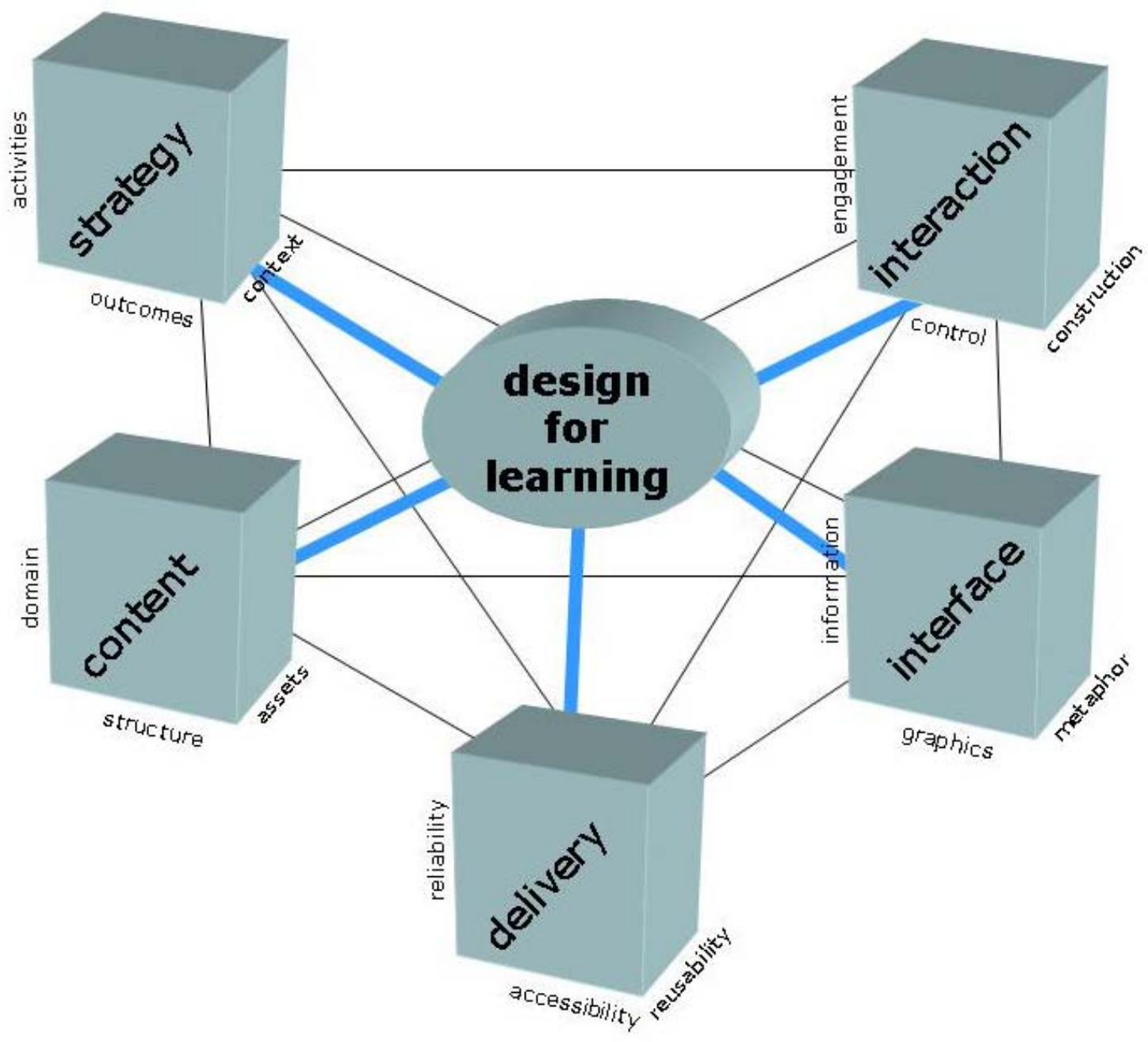

Figure 1: Design for Learning Model

\section{Conclusion}

Through an appraisal of current use of the instructional design process and the potential of adopting proactive models and interactive metrics, this paper has argued that online teaching and learning must go beyond delivery to achieve the potential of interactive and collaborative networks of learners. The design and implementation of effective online learning environments must appreciate that delivery is not the only or primary aim.

Do we need emancipated learners? In the context of online learning and reflective practice I answer yes, because only then can we realise the dynamics that asynchronous and synchronous communications enable. Independence from specific times or places is one characteristic of the new generation of learners, and this is likely to significantly impact the way education opportunities are provided and accessed.

Do we need instructional design? As traditionally practiced, I answer no. However, we continue to need a comprehensive understanding of how people learn and the way learners can best be engaged in online environments. Where conventional instructional design models and process fail to effectively address these factors, we need to consider new models that integrate the pedagogies of online, learner-centred environments. 
We are at the crossroads. Do we maintain the traditional standards of instructional design or do we take a risk? Will teachers choose to work with emancipated and empowered learners, or will they adopt a conventional teacher-centred and controlling role? Will learners accept a passive, information transmission role or will they be enraged if not engaged?

It will remain our choice as to how far we are prepared to go, and how much we are prepared to risk, to fully realise the potential of online teaching and learning.

\section{References}

Driscoll, M.P. (2005). Psychology of learning for instruction. ( $3^{\text {rd }}$ ed.). Boston, MA: Routledge.

Kennedy, G. (2004). Promoting cognition in multimedia interactivity research. Journal of Interactive Learning Research, 15(1), 43-61.

Laurel, B. (1991). Computers as theatre. Reading, MA: Addison Wesley.

Laurillard, D. (1993). Rethinking university teaching: A framework for the effective use of educational technology. London, UK: Routledge.

Moore, M.G. (1989). Editorial: Three types of interaction. The American Journal of Distance Education, 3(2), 1-7.

Prensky, M. (2005). Engage me or enrage me: What today's learners demand. Accessed from http://www.educause.edu/ir/library/pdf/erm0553.pdf, $8^{\text {th }}$ February 2006.

Reigeluth, C.M. (1999). Instructional-design theories and models. (Vol. II). Mahwah: Erlbaum.

Rourke, L., Anderson, T. Garrison, D. R., \& Archer, W. (1999). Assessing social presence in asynchronous, text-based computer conferencing. Journal of Distance Education, 14(3), 5170.

Sims, R. (1997a). Interactive learning as an "emerging” technology: A reassessment of interactive and instructional design strategies. Australian Journal of Educational Technology, 13(1), 6884.

Sims, R. (1997b). Interactivity: A Forgotten Art? Computers in Human Behavior, 13(2), 157-180.

Sims, R. (1999). Interactivity on stage: Strategies for learner-designer communication. Australian Journal of Educational Technology, 15(3), 257-272.

Sims, R. (2003). Interactivity and feedback as determinants of engagement and meaning in elearning environments, in S. Naidu (Ed) Learning \& Teaching With Technology: Principles and Practices. London, UK: Kogan Page.

Sims, R., Dobbs, G. \& Hand, T. (2002). Enhancing quality in online learning: Scaffolding design and planning through proactive evaluation. Distance Education, 23(2). 135-148.

Sims, R. \& Bovard, B. (in press). Using elaborations of online presence to foster critical thinking and reflection, in M. Spector (Ed.). Finding your online voice: Stories told by experienced online educators. Lawrence Erlbaum.

Sims, R. \& Hedberg, J. (in press). Encounter theory: A model to enhancing online communication, interaction and engagement, in C. Jawah (Ed) Interactions in Online Education: Implications for Theory and Practice. London, UK: Routledge Education.

Sims, R. \& Jones, D. (2003). Where practice informs theory: Reshaping instructional design for academic communities of practice in online teaching and learning. Information Technology, Education and Society, 4(1), 3-20.

Ulmer, G.L. (2003). Internet invention: From literacy to electracy. New York: Longman.

Wagner, E.D. (1994). In support of a functional definition of interaction. The American Journal of Distance Education, 8(2), 6-29.

Wikipedia (2006a). Instruction. Accessed from http://en.wikipedia.org/wiki/Instruction, February $8^{\text {th }} 2006$.

Wikipedia (2006b). Design. Accessed from http://en.wikipedia.org/wiki/Design, February $8^{\text {th }}$ 2006.

Wikipedia (2006c). Instructional Design. Accessed from http://en.wikipedia.org/wiki/Instructional_design, February $8^{\text {th }} 2006$. 
Please cite as: Sims, R. (2006). Beyond instructional design: Making learning design a reality. Journal of Learning Design, 1(2), 1-7. http://www.jld.qut.edu.au/ 